\title{
Determination of scrub typhus in negative patients for dengue and rickettsia
}

\author{
DOI: $10.46932 / s f j d v 2 n 1-063$
}

Received in: November 1st, 2020

Accepted in: December 30th, 2020

\author{
MC. Manuel Antonio Castillo Polanco \\ Unidad Interinstitucional de Investigación Clínica y Epidemiológica \\ Facultad de Medicina de la Universidad Autónoma de Yucatán \\ Mérida, Yucatán, México \\ E-mail: manuelcastillo@outlook.com \\ MC. Luis Enrique de la Cruz Burgoa \\ Unidad Interinstitucional de Investigación Clínica y Epidemiológica \\ Facultad de Medicina de la Universidad Autónoma de Yucatán \\ Mérida, Yucatán, México \\ E-mail: dlcb.enrique@gmail.com \\ Dr. Gaspar Fernando Peniche Lara \\ Unidad Interinstitucional de Investigación Clínica y Epidemiológica \\ Facultad de Medicina de la Universidad Autónoma de Yucatán \\ Mérida, Yucatán, México \\ E-mail: gaspar.peniche@ correo.uady.mx
}

\section{RESUME}

The scrub typhus is an endemic disease from Asia caused by a bacteria called Orientia tsutsugamushi which is characterized by the presence of general discomfort, headache, body aches and muscle pain, fever, rash, and enlarged lymph nodes, similar to symptoms of many other diseases as dengue or rickettsia. In Latin America the presence of $\mathrm{O}$. tsutsugamushi has already been reported; nevertheless, as in México the presence of this bacteria has not been detected, is not considered as a part of molecular diagnosis consequently we decided to identify the presence of clinical cases of scrub typhus in negative patients for dengue and rickettsia. The sample was established by 21 patients from public and private hospitals of Yucatán from January until June of 2017, under suspicion of dengue and rickettsia but serologically negative in these. The DNA was extracted and amplified for the gen STA-56, it showed a band in level between 300-400 pb where there was the positive control. With these results through the PCR exists the possibility of having positive cases of $\mathrm{O}$. tsutsugamushi; however, for its low quality in the visualization, those should be confirmed by genetic sequencing.

Keywords: Orientia tsutsugamushi, dengue, rickettsia, polymerase chain reaction, negative diagnosis, febrile illness, rash.

\section{INTRODUCTION}

Scrub typhus is an acute febrile illness transmitted by vector ${ }^{1}$. His agent, Orientia tsutsugamushi, is an intracellular bacteria transmitted by the sting of mites of the genus leptotrombidium associated principally with rodents of the genus Rattus (common rat) ${ }^{2}$.

The Rickettsiacea family includes two genus, the genus Rickettsia and the genus Orientia. This 
phylogenetic relation is the cause of the infections produced by Rickettsia and Orientia ${ }^{3}$ share a very similar clinical symptoms between them, giving like consequent the typical manifestations of high fever, general discomfort, headache, body aches, muscle pain, rash, and enlarged lymph nodes ${ }^{4}$.

Scrub typhus is an endemic disease from Asia ${ }^{5}$. Until 2006, infections caused by Orientia had not been confirmed beyond Asia and the Pacific; however, in that year were two cases of infection reported with Orientia species in the Middle East and Chile ${ }^{1}$. Land, sea, and air trade, together with global mobility, may be responsible for the spread of these endemic diseases across the globe.

In Mexico, no cases of Orientia infection have been reported until the date ${ }^{6}$; however, in the state of Yucatan, a number of suspicious cases have been detected in the hospital admission with the clinical manifestations of rash fever, where the first diagnosis impressions, dengue and rickettsia, have been previously ruled out by the main diagnostic tests. The clinical records of these patients and the environment in which they lives indicate the coexistence with vectors and ectoparasites that could suggest, starting from the antecedent in Chile, a possible infection by O. tsutsugamushi, explaining in this way the similarity of the clinical manifestations with rickettsiosis ${ }^{7}$ and the negativity of the laboratory tests; reason why we took on the task of identifying, through DNA tests, the presence of $O$. tsutsugamushi in the blood samples of these patients.

\section{MATERIALS AND METHODS}

This cross-sectional study was carried between January 2017 and June 2017 in the Inter-Institutional Unit of Clinical and Epidemiological Research (IIUCE) belonging to the Faculty of Medicine of the Autonomous University of Yucatan, in the city of Merida, Yucatan, Mexico.

\subsection{SAMPLE COLLECTION}

A total of 21 blood samples were recollected from patients with symptoms suggestive of rash fever who gave negative results to the dengue and rickettsia tests and which were collected during the period from January to June 2017 in the city of Merida, Yucatan. These patients were selected from the General Hospital "Dr. Agustín O'Horán", the "ISSSTE Regional Mérida" and private hospitals referred to the InterInstitutional Unit of Clinical and Epidemiological Research (IIUCE) for molecular diagnosis.

\subsection{DNA EXTRACTION}

DNA was extracted from each of the samples using the Zymoclean ${ }^{\mathrm{TM}}$ Gel DNA Recovery Kit and following the established protocol ${ }^{8}$, obtaining a total of $50 \mu 1$ of DNA for each blood sample. 


\subsection{MOLECULAR DIAGNOSIS BY END-POINT PCR}

Once the DNA was extracted, polymerase chain reaction (PCR) was performed with the aim of amplifying a common region of gene for all $O$. tsutsugamushi species, being chosen as the STA-56 gene' For each diagnosis sample was used $12.5 \mu \mathrm{l}$ of DreamTaq Green Polymerase, $0.25 \mu \mathrm{l}$ of the first Pr1 (5 TAC ATT AGC TGC AGG TAT GAC-3) and 0.25 $\mu 1$ of the first Pr2 (5 -AAT TCT TCA ACC AAG CGA TCC-3 $)^{10}$. Subsequently $7 \mu 1$ of nuclease-free water was added, finally, $5 \mu 1$ of the DNA extracted from the respective sample was added, making a total of $25 \mu$ for each diagnosis. 21 diagnostic samples and 2 controls were prepared, one negative using $5 \mu 1$ nuclease-free water instead of $5 \mu 1$ diagnostic DNA and one positive using $4 \mu 1$ nuclease-free water and $1 \mu 1$ pure $O$. tsutsugamushi DNA.

The 23 samples were processed by the MULTIGENE thermocycler under standardized conditions for 160 minutes. Once the PCR was completed, the products obtained underwent electrophoresis in $1.5 \%$ agarose gel under the conditions of $80 \mathrm{~V}$ for 90 minutes. At the end of this process, the agarose gel was immersed in ethidium bromide (EtBr) for 10 minutes; subsequently was washed with a water distiller for another 10 minutes. Once the bath with the distilled water was finished, the gel was developed in the Gel Doc XR (Bio-Rad) system using UV light and capturing the image using computer software.

\section{RESULTS}

After analyzed them by UV light, one band was detected at the position of $317 \mathrm{bp}$ in 4 of the 21 samples, just where the positive control of the Orientia tsutsugamushi STA-56 gene was located (Figure 1); the negative control as the remaining samples no showed visual data of any band presence.

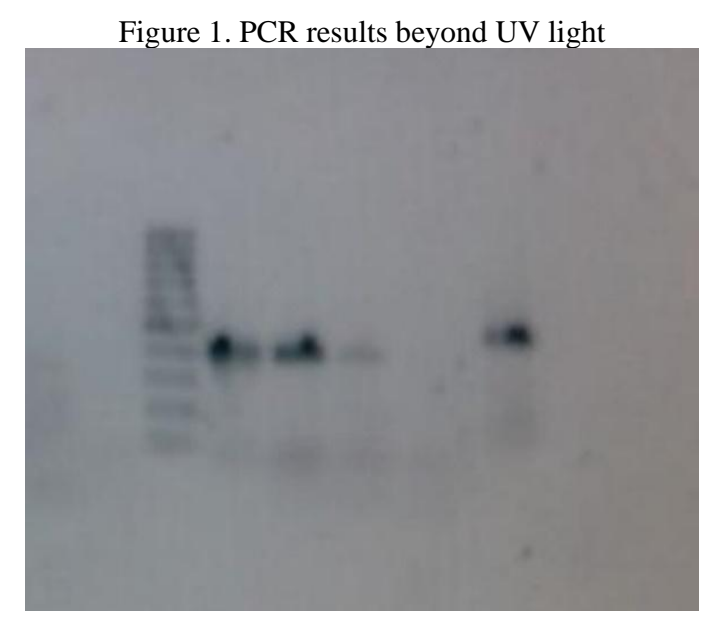

The analysis of the reference notes and clinical records of the four patients with molecular evidence of Orientia show us that all patients presented fever $>39^{\circ} \mathrm{C}$ with at least 7 days of evolution. The four patients presented asthenia, adynamia, general discomfort, nausea, myalgia, arthralgias, and maculopapular rash. In two patients hyperbilirubinemia was found with direct pattern exteriorizing jaundice in skin and conjunctiva. 
In the physical examination, hepatomegaly was found only in one patient. Complete blood count found leukocytosis in all four patients. Abnormalities in coagulation like PT prolongation and thrombocytopenia were found in two patients. In only one patient was death recorded days after the blood extraction.

\section{DISCUSSION}

In the present study, the PCR test was directed at the $59 \mathrm{kDa}$ gene, which is a specific protein exclusive to Orientia tsutsugamushi. This gene has four domains that are hypervariable responsible for antigenic variation. ${ }^{9}$

In our study the visualization of the band in the mentioned samples was only for the contrast adjust through the software since at first glance was difficult visualize, in such a way we can not conclude that bands belongs to STA-56 gen but neither we can discard it, since electrophoresis clearly produces a product of similar size between 300-400 bp.

There are many factors that have influenced this phenomenon, the DNA quality analyzed, the researcher technique for the PCR or it could have been variations in the numbers of base pairs between the diagnostics and the STA-56 gen proportioned by the GenBank.

Guo HB et al (1997) mentioned that PCR amplification of O. tsutsugamushi from the blood often lacks sensitivity because hemoglobin, porphyrin and other factors can inhibit PCR, although it is possible to obtain and process blood to avoid inhibitors ${ }^{11}$; on the other hand, it has been noted that a large number of positive serological cases were negative for PCR, which may indicate a low sensitivity of this test, either due to the low copy numbers or the excess of the DNA of the host as various studies established ${ }^{12,13}$, this could be the reason why visualize the bands was difficult.

\section{CONCLUSION}

Despite the low quality of the visualization, in patients with negative results for dengue and rickettsia, we concluded that there exists the possibility of having positive cases of scrub fever by the results of the PCR since we can observe the band between 300-400 pb, matching with the clinical symptoms and laboratory patterns of infection by O. tsutsugamushi. We suggest another test of detection with the blood samples including a sequence by Sanger method to verify the percent of similitude of the obtained products compares with $O$. tsutsugamushi data from GenBank.

If confirmed by a sequencing method, the first molecular evidence of the disease would be established in the State of Yucatan, in order to integrate and consider it within vector-borne diseases, thus establishing a timely diagnosis and treatment, as well as directing measures towards primary prevention. 


\section{BIBLIOGRAPHIC REFERENCES}

1. Weitzel, T., Dittrich, S., López, J., Phuklia, W., Martinez-Valdebenito, C., Velásquez, K., Blacksell, S. D., Paris, D. H., \& Abarca, K. (2016). Endemic Scrub Typhus in South America. The New England journal of medicine, 375(10), 954-961. https://doi.org/10.1056/NEJMoa1603657

2. Paris, D. H., Shelite, T. R., Day, N. P., \& Walker, D. H. (2013). Unresolved problems related to scrub typhus: a seriously neglected life-threatening disease. The American journal of tropical medicine and hygiene, 89(2), 301-307. https://doi.org/10.4269/ajtmh.13-0064

3. Boone, D., Castenholz, R. and Garrity, G., 2005. Bergey's manual of systematic bacteriology. 4th ed. New York: Springer, p.96.

4. Ogawa, M., Hagiwara, T., Kishimoto, T., Shiga, S., Yoshida, Y., Furuya, Y., Kaiho, I., Ito, T., Nemoto, H., Yamamoto, N., \& Masukawa, K. (2002). Scrub typhus in Japan: epidemiology and clinical features of cases reported in 1998. The American journal of tropical medicine and hygiene, 67(2), 162-165. https://doi.org/10.4269/ajtmh.2002.67.162

5. Watt, G., \& Parola, P. (2003). Scrub typhus and tropical rickettsioses. Current opinion in infectious diseases, 16(5), 429-436. https://doi.org/10.1097/00001432-200310000-00009

6. Boletin Epidemiológico de la semana 39. Sistema Naciona de Vigilancia Epidemiológica (2017). Recuperado el 22 Junio 2017, desde https://www.gob.mx/cms/uploads/attachment/file/261953/sem39.pdf

7. Paris, D. H., \& Dumler, J. S. (2016). State of the art of diagnosis of rickettsial diseases: the use of blood specimens for diagnosis of scrub typhus, spotted fever group rickettsiosis, and murine typhus. Current opinion in infectious diseases, 29(5), 433-439. https://doi.org/10.1097/QCO.0000000000000298

8. Zymo Research Protocol. Zymoresearch.com. (2017). Recuperado el 28 Junio 2017, desde http://www.zymoresearch.com/downloads/dl/file/id/34/d4001i.pdf.

9. Zheng, L., Bi, Z., Kou, Z., Yang, H., Zhang, L., \& Zhao, Z. (2015). Genotype diversity and distribution of Orientia tsutsugamushi in scrub typhus patients and rodents in Shandong, northern China. Infection, genetics and evolution : journal of molecular epidemiology and evolutionary genetics in infectious diseases, 36, 126-130. https://doi.org/10.1016/j.meegid.2015.09.008

10. Liu, Y. X., Cao, W. C., Gao, Y., Zhang, J. L., Yang, Z. Q., Zhao, Z. T., \& Foley, J. E. (2006). Orientia tsutsugamushi in eschars from scrub typhus patients. Emerging infectious diseases, 12(7), 1109-1112. https://doi.org/10.3201/eid1207.050827

11. Guo HB, Pan SZ, Li XF, Tang JQ, Zhang Y, Wu GH. (1997). Studies on detecting and typing of Rickettsia tsutsugamushi by polymerase chain reaction. Chinese Journal of Zoology.13(2), 138-11

12. Srinivasan, S., \& Menon, T. (2017). Molecular detection of Orientia tsutsugamushi from suspected scrub typhus cases. Indian journal of pathology \& microbiology,60(1), 70-73. https://doi.org/10.4103/0377-4929.200043

13. Sonthayanon, P., Chierakul, W., Wuthiekanun, V., Blacksell, S. D., Pimda, K., Suputtamongkol, Y., Pukrittayakamee, S., White, N. J., Day, N. P., \& Peacock, S. J. (2006). Rapid diagnosis of scrub typhus in rural Thailand using polymerase chain reaction. The American journal of tropical medicine and hygiene, 75(6), 1099-1102. 